\title{
Co-trimoxazole Resistance in Clinical Isolates of Stenotrophomonas maltophilia
}

\author{
Neha Shah ${ }^{1 *}$, S. Basireddy ${ }^{2}$, Sreeja Vamsi ${ }^{2}$ and Vasanti Kabra ${ }^{2}$ \\ ${ }^{1}$ Department of Microbiology, Smth Kashibai Navale Medical College and General \\ Hospital, Pune, India \\ ${ }^{2}$ Department of Microbiology, SVS Medical College, Mehboobnagar, A.P., India \\ *Corresponding author
}

\section{Keywords}

Stenotrophomonas maltophilia, Cotrimoxazole, NFGNB, Drug resistance

Article Info

Accepted:

04 January 2018

Available Online:

10 February 2018

\section{A B S T R A C T}

Stenotrophomonas maltophilia is increasingly being isolated as a nosocomial pathogen. It is the third most common non-fermenting gram negative bacilli (NFGNB) isolated from the clinical samples especially from the respiratory specimens, blood, urine and pus samples. Though inherently resistant to many groups of antibiotics, its susceptibility to co-trimoxazole is a peculiar character, because of which Co-trimoxazole is considered as the drug of choice for treating these infections. Recently there has been an increase in the incidence of resistance against Co-trimoxazole with variable resistance towards other antibiotics. In the present study $14.2 \%$ of the isolates were resistant to Co-trimoxazole. Resistant to other antibiotics was also very high. Ciprofloxacin and Chloramphenicol have shown reasonably good activity against this organism making these drugs as therapeutic alternatives in Co-trimoxazole resistant cases

\section{Introduction}

Stenotrophomonas maltophilia is a motile, aerobic, non-fermenting, Gram-negative bacillus. It is isolated from various sources in nature like water, soil, plants, and animals. $S$. maltophilia is an important opportunistic human pathogen, causing nosocomial infections especially in immunocompromised patients. It is the third most common pathogenic non-fermenting gram negative bacilli (NFGNB) worldwide after Pseudomonas aeruginosa and Acinetobacter calcoaceticus -baumannii complex (Looney et al., 2009, LiPuma et al., 2007). S. maltophilia infections include pneumonia, bloodstream infections, urinary tract infections, soft tissue infections, meningitis, and ocular infections.

This pathogen is characterized by intrinsic resistance to multiple classes of antibiotics, due to various mechanisms such as decreased permeability, production of b-lactamases and of aminoglycoside modifying enzymes, or the presence of multidrug efflux pumps (Sanchez et al., 2009).

For a routine laboratory it is always a difficult 
task to identify the NFGNB up to the species level, because of which these organisms are frequently under reported. Inherently multidrug resistance nature including resistance to carbapenems necessitates the appropriate identification of this organism and determining the susceptibility patterns. The present study aims at isolating, identifying and determining the susceptibility patterns of Stenotrophomonas maltophilia from various clinical specimens in our hospital.

\section{Materials and Methods}

Routine bacterial cultures were performed for various samples received in the department of microbiology during the study period of January 2013 to Dec 2014. All the culture positive organisms were identified based on routine biochemical tests.

All the oxidase negative non-fermenting gram negative bacilli (NFGNB) were processed further by performing additional tests like lysine decarboxylase tests, esculin hydrolysis, OF maltose, OF mannitol and pigment production to identify Stenotrophomonas maltophilia species(Winn et al., 2006).

Antimicrobial susceptibility testing was performed by the Kirby-Bauer disc diffusion method. The agents tested against Stenotrophomonas included ceftazidime $(30 \mu \mathrm{g}), \quad$ tigecycline $(15 \quad \mu \mathrm{g})$, piperacillin/tazobactum $\quad(100 / 10 \quad \mu \mathrm{g})$, cefaperazone sulbactum $(75 / 10 \mu \mathrm{g})$, amikacin (30 $\mu \mathrm{g})$, chloramphenicol $(30 \mu \mathrm{g})$, ciprofloxacin $(5 \mu \mathrm{g})$, doxycycline $(30 \mu \mathrm{g})$, andtrimethoprim/ sulfamethoxazole $(1.25 / 23.75 \mu \mathrm{g})$, imipenem $(10 \mu \mathrm{g})$. The results were interpreted according to the Clinical and Laboratory Standards Institute (CLSI) criteria (Wanye, 2011). For the agents where specific CLSI criteria for $S$. maltophilia are not available, the relevant criteria for nonEnterobacteriaceae were used. Escherichia coli ATCC 25922 and Pseudomonas aeruginosa ATCC 27853 were used as quality control strains

\section{Results and Discussion}

A total of 297 oxidase negative nonfermenting gram negative bacilli were isolated from various clinical samples during the study period, out of which $21(7 \%)$ were identified as Stenotrophomonas maltophilia species.

Majority of the Stenotrophomonas were isolated from respiratory specimens $(57.1 \%)$ followed by pus samples $(33.3 \%)$ and blood $(9.5 \%)$. Males were predominantly affected $(71.4 \%)$ followed by females $(28.6 \%)$ and all these organisms were isolated in the age group of 27 years to 65 years.

All the isolates were resistant to imipenem and ceftazidime (100\%).Co-trimoxazole resistance was observed in $3(14.2 \%)$ out of the 21 isolates. Ciprofloxacin and chloramphenicol resistance was observed in 6(28.5\%) isolates each and doxyxycline resistance in $7(33.3 \%)$ isolates.

Other antibiotics like cefaperazone sulbactum and piperacillin tazobactum were resistant in half of the isolates (47.6\% each). Amikacin resistance was quiet high with 12 out of the 21 isolates $(57.1 \%)$ being resistant to it. The least resistance was observed for tigecycline with all the isolates being susceptible to it $(0 \%$ resistance)

S. maltophilia is an opportunistic multidrug resistant (MDR) Gram-negative bacterium that causes serious infection in immunocompromised patients. Though is not a highly virulent pathogen, it is recognized as a significant nosocomial pathogen and the incidence of these infections in the hospitals are on rise.

This lysine-positive NFGNBs is having a 
characteristic contrasting susceptibility pattern to that of $P$. aeruginosa showing sensitivity to co-trimoxazole and resistance towards imipenem. Co-trimoxazole has long been considered as an effective drug for the treatment of these infections, but there has been an increase in antimicrobial resistance of $S$. maltophilia over recent years to this drug which was once a preferred treatment for $S$. maltophilia infections (Gautam et al., 2009; Goldberg et al., 2012). Resistance to cotrimoxazole, over imposed on the inherent multidrug resistance nature to many other antibiotics like aminoglycosides, beta lactams, cephalosporins, carbapenems and many fluoroquinolones makes this organism as an extremely difficult pathogen for the treatment.

Antimicrobial susceptibility testing methods of $S$. maltophilia are also not clearly standardized, and there is poor correlation between disk diffusion and agar dilution results for some agents (Sader, 2005). The European Committee on Antimicrobial Susceptibility Testing (EUCAST) and the British Society for Antimicrobial Chemotherapy (BSAC) report clinical breakpoint data only for trimethoprimsulfamethoxazole whereas Clinical and Laboratory Standards Institute (CLSI) recommends dilution testing for trimethoprimsulfamethoxazole, ceftazidime, chloramphenicol, levofloxacin, minocycline, and ticarcillin-clavulanate, and disk diffusion testing for only trimethoprimsulfamethoxazole, levofloxacin, and minocycline. [EUCAST, 2010, BSAC, 2010] Inspite of the lack of the in vitro guidelines many other antibiotics have been used therapeutically either alone or in combination therapy (Falagas et al., 2008)

In the present study resistance to cotrimoxazole was observed in $14 \%$ of the total isolates which is in accordance with the study conducted by Samonis et al., (Samonis et al., 2012) where $13.2 \%$ of the isolates were resistant to this antibiotic. In a 2012 study of S. maltophilia recovered from cystic fibrosis patients, $24.2 \%$ of the patients had TMXresistant isolates (Milne et al., 2012). The SENTRY Antimicrobial Surveillance Program reported a global resistance rate of $4.7 \%$ (1997-2003) (Sader, 2005). This is in contrast with a Taiwanese study of 103 S. maltophilia isolates from hospitalized patients, which demonstrated as high as $25 \%$ trimethoprimsulfamethoxazole resistance (Chang et al., 2007).

Fig.1 Resistance pattern of Stenotrophomonas to various antibiotics

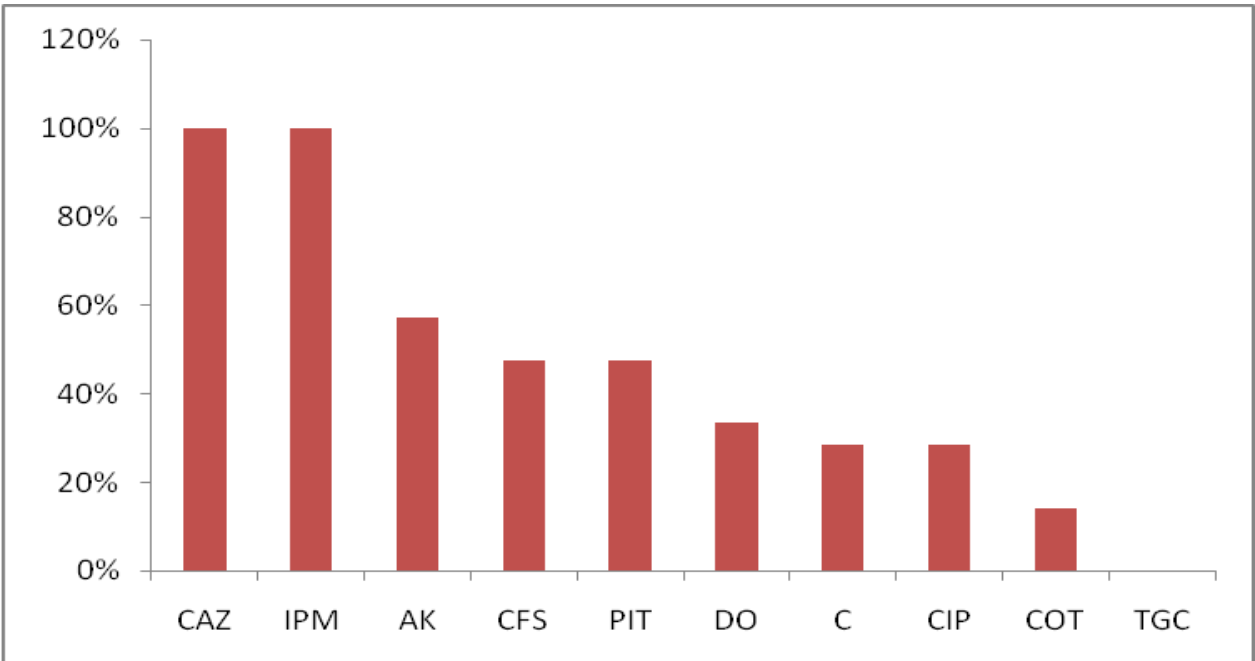


In another study conducted by Arora et al., (2012) in India, it was observed that over a period of years there was a steady increase in the resistance of Stenotrophomonas to cotrimoxazole from 9 to $30 \%$ of the isolates (Arora et al., 2012).

In general resistance rates to trimethoprimsulfamethoxazole have been reported to vary geographically (Livermore et al., 2008, Farrell et al., 2010), but were usually less than $20 \%$.In our study also similar results were observed. Development of resistance to trimethoprim-sulfamethoxazole has been reported owing to modified target genes sull and sul2. The association of these genes with the mobile genetic elements is of significant concern because of the probability of wide spreading of this resistance (Toleman et al., 2007)

In the present study all the isolates were resistant to imipenem which is expected, as this organism is inherently resistant to imipenem. Though routine susceptibility testing with imipenem is not recommended, observing resistance to this drug in routine sensitivity plates gives us a clue towards identification of this organism. Apart from imipenem, this organism also has shown absolute resistance against ceftazidime in our study. High rates of ceftazidime resistance have been reported by many authors. Samonis et al., reported a resistance rate of $73.5 \%$ for the ceftazidime. Milne et al., reported that $90 \%$ of the Stenotrophomonas isolates were resistant to ceftazidime. EUCAST report $S$. maltophilia to be intrinsically resistant to ceftazidime, regardless of the result of susceptibility testing.

Amikacin resistance was also considerably high in the present study with $52 \%$ of the isolates being resistant. This is similar to betriu et al., (Betriu et al., 2001) study where $62.7 \%$ of the isolates were resistant to amikacin. In Milne et al., (2012) study 86\% of the isolates were resistant to amikacin. In contrast Samonis et al., (2012) observed very high sensitive rate to this antibiotic where $82.4 \%$ of the isolates were susceptible to amikacin.

Tigecycline has shown the least resistance in the present study with all the isolates being susceptible to it. Only few studies are available in the literature regarding the tigecycline susceptibility patterns against Stenotrophomonas. In an antimicrobial susceptibility study of 1,586 S. maltophilia clinical isolates recovered from medical centers in Europe, North America, Asia and the Pacific region and Latin America, tigecycline was reported as the most effective antibiotic with more than $90 \%$ of the isolates being susceptible to this drug irrespective of geographical regions (Farrell et al.,2010). Tigecycline is a newer antibiotic with only a limited clinical knowledge and evidence regarding the usefulness of this drug in the treatment of Stenotrophomonas infections. Though in vitro susceptibility testing showed fruitful results, it cannot be attributed fully to in vivo situations where pharmacokinetics and pharmacodynamic properties play a significant role. Above this, the high cost of this drug also limits its extensive usage in countries like India. Further research needs to be carried out before fully adopting this agent as a therapeutic alternative.

Apart from tigecycline and cotrimoxazole, in the present study ciprofloxacin and chloramphenicaol have also shown good in vitro activity with only $28.5 \%$ of the isolates being resistant to these antibiotics. The resistance rates to these two drugs varied from study to study. In Betriu et al., (2001) study the resistant to ciprofloxacin and chloramphenicol was $68.7 \%$ and $39.3 \%$ respectively where as in Samonis et al., (2012) study the resistance rate was $17.6 \%$ 
and $16.2 \%$ respectively. In Arora et al., (2012) study only $16 \%$ of the isolates were resistant to fluoroquinolones.

Doxycycline followed ciprofloxacin with $33.3 \%$ resistance which is intermediate between the findings of Milne et al., (2012) and Arora et al., (2012) study where $13.7 \%$ and $59 \%$ of the isolates were resistant to this antibiotic respectively. Other antibiotics live piperacillin/tazobactum and cefaperazone/ sulbactum have shown equivocal resistance patterns allowing their utility in only half of the isolates (Fig. 1).

The present study highlights the susceptibility patterns of the Stenotrophomonas against various antimicrobial agents. Bacteria can develop resistance to any antibiotic in due course of time especially under the intense antibiotic pressure in the hospital setup. Thorough understanding of the local susceptibility patterns along with proper infection control measures will help in the appropriate management of patients and further prevention of the spread of these resistant infections.

To conclude, resistance has been observed in few isolates against trimethoprimsulfamethoxazole. Still this drug showed good in vitro activity against majority of the $S$. maltophilia isolates. Ciprofloxacin and doxycycline can be considered as an effective alternatives especially in co-trimoxazole resistant infections or when the patients are allergic to co-trimoxazole. Though tigecycline is the highly effective antibiotic in the present study, high cost of this drug and limited clinical experience regarding the effectiveness of this antibiotic against Stenotrophomonas prevents its wider usage.

\section{References}

Arora S, Gautam V, Ray P. Changing susceptibility patterns of non-fermenting
Gram-negative bacilli. Indian Journal of Medical Microbiology 2012; 30(4):48586.

Betriu C, Sanchez A, Palau ML, Carlos S. Antibiotic resistance surveillance of Stenotrophomonas maltophilia. J Antimicrob Chemother. 2001; 48: 152154.

Chang LL, Lin HH, Chang CY, Lu PL. Increased incidence of class 1 integrons in trimethoprim/sulfamethoxazole-resistant clinical isolates of Stenotrophomonas maltophilia. J. Antimicrob. Chemother. 2007; 59(5):1038-1039.

Clinical and Laboratory Standards Institute. Performance standards for antimicrobial susceptibility testing; twenty-first informational supplement, M100-S21. Wanye, PA: Clinical and Laboratory Standards Institute; 2011.

European Committee on Antimicrobial Susceptibility Testing www.eucast.org/ fileadmin/src/media/PDFs/EUCAST_files /Disk_test_documents/EUCAST_breakpo ints_v1.1.pdf (Accessed 19 December 2010)

Expert Rules in Antimicrobial Susceptibility Testing www.escmid.org/fileadmin/src/ media/PDFs/4ESCMID_Library/3Publica tions/EUCAST_Documents/Other_Docu ments/EUCAST_Expert_rules_final_Apri 1_20080407.pdf (Accessed 19 December 2010)

Falagas, M. E., P. E. Valkimadi, Y. T. Huang, D. K. Matthaiou, and P. R. Hsueh. 2008. Therapeutic options for Stenotrophomonas maltophilia infections beyond co-trimoxazole: a systematic review. J. Antimicrob. Chemother. 62:889-894.

Farrell DJ, Sader HS, Jones RN. Antimicrobial susceptibilities of a worldwide collection of Stenotrophomonas maltophilia isolates tested against tigecycline and agents commonly used for $S$. maltophilia infections. Antimicrob Agents Chemother 2010; 54:2735-7.

Farrell DJ, Sader HS, Jones RN. Antimicrobial susceptibilities of a worldwide collection 
of Stenotrophomonas maltophilia isolates tested against tigecycline and agents commonly used for $S$. maltophilia infections. Antimicrob. Agents Chemother. 2010; 54(6):2735-2737.

Gautam V, Ray P, Vandamme P, Chatterjee SS, Das A,Sharma K, et al., Identification of lysine positive non-fermenting gram negative bacilli (Stenotrophomonas maltophilia and Burkholderia cepacia complex). Indian J Med Microbiol 2009; 27:128-33.

Goldberg E, Bishara J. Contemporary unconventional clinical use of cotrimoxazole. Clin. Microbiol. Infect. 2012; 18(1):8-17.

LiPuma JJ, Currie BJ, Lum GD, Vandamme PA. Burkholderia, Stenotrophomonas, ralstonia, cupriavidus, pandoraea, brevundimonas, comamonas and acidovorax. Chapter 9. In: Manual of clinical microbiology. 9th ed. Murray PR, Baron EJ, Jorgensen JH, Landry ML, Pfaller MA, editors. Washington, DC: ASM Press; 2007; 749-69.

Livermore DM, Hope R, Brick G, Lillie M, Reynolds R; BSAC Working Parties on Resistance Surveillance. Nonsusceptibility trends among Pseudomonas aeruginosa and other non-fermentative Gram-negative bacteria from bacteraemias in the UK and Ireland, 2001-06. J Antimicrob Chemother 2008; 62(2):55-63.

Looney WJ, Narita M, Mu“hlemann K. Stenotrophomonas maltophilia: an emerging opportunist human pathogen. Lancet Infect. Dis. 2009; 9(5): 312-323.

Methods for Antimicrobial Susceptibility Testing. Version 9.1. March 2010. British
Society for Antimicrobial Therapy www.bsac.org.uk/Resources/BSAC/ Version_9.1_March_2010_final.pdf (Accessed 19 December 2010)

Milne KEN, Gould IM. Combination antimicrobial susceptibility testing of multidrug-resistant Stenotrophomonas maltophilia from cystic fibrosis patients. Antimicrob. Agents Chemother. 2012; 56(8):4071-4077

Sader HS, Jones RN. Antimicrobial susceptibility of uncommonly isolated non-enteric Gram-negative bacilli. Int. J. Antimicrob. Agents 2005; 25(2):95-109.

Samonis G, Karageorgopoulos DE, Maraki S, Levis P, Dimopoulou D. Stenotrophomonas maltophilia Infections in a General Hospital: Patient Characteristics, Antimicrobial Susceptibility, and Treatment Outcome. PLoS ONE 2012; 7(5): 1-7.

Sanchez MB, Herna'ndez A, Martı'nez JL. Stenotrophomonas maltophilia drug resistance. Future Microbiol. 2009; 4(6): 655-660.

Toleman MA, Bennett PM, Bennett DM, Jones RN, Walsh TR. Global emergence of trimethoprim/sulfamethoxazole resistance in Stenotrophomonas maltophilia mediated by acquisition of sul genes. Emerg. Infect.Dis. 13(4): 559-565 (2007).

Winn W, Allen S, Jande W, Koneman E, Procop G, Schrekernbenger P, Woods G. Koneman's color atlas and textbook of diagnostic microbiology. 6th ed. Baltimore, USA: Lippincott Williams and Wilkins Publishers; 2006; Pp. 303-91.

\section{How to cite this article:}

Neha Shah, S. Basireddy, Sreeja Vamsi and Vasanti Kabra. 2018. Co-trimoxazole Resistance in Clinical Isolates of Stenotrophomonas maltophilia. Int.J.Curr.Microbiol.App.Sci. 7(02): 120125. doi: https://doi.org/10.20546/ijcmas.2018.702.015 